\title{
Evaluation of Antibiotic Resistance and Biofilm Production among Clinical Strain Isolated from Medical Devices
}

\author{
Veronica Folliero $\left(D,{ }^{1}\right.$ Gianluigi Franci $\left(D^{2},{ }^{2}\right.$ Federica Dell'Annunziata, ${ }^{1}$ Rosa Giugliano $(i)$, \\ Francesco Foglia $\mathbb{D},{ }^{1,3}$ Rossella Sperlongano, ${ }^{1}$ Anna De Filippis $\mathbb{D}^{1},{ }^{1}$ Emiliana Finamore, ${ }^{1,3}$ \\ and Massimiliano Galdiero $\mathbb{D}^{1,3}$
}

\author{
${ }^{1}$ Department of Experimental Medicine, University of Campania "Luigi Vanvitelli", Naples 80138, Italy \\ ${ }^{2}$ Department of Medicine Surgery and Dentistry "Scuola Medica Salernitana", University of Salerno, Fisciano 84081, Italy \\ ${ }^{3}$ U.O.C. of Virology and Microbiology, A.O.U. University of Campania "Luigi Vanvitelli", Naples 80138, Italy
}

Correspondence should be addressed to Massimiliano Galdiero; massimiliano.galdiero@unicampania.it

Veronica Folliero and Gianluigi Franci contributed equally to this work.

Received 31 May 2021; Revised 9 July 2021; Accepted 6 August 2021; Published 14 August 2021

Academic Editor: Joseph Falkinham Copyright $\odot 2021$ Veronica Folliero et al. This is an open access article distributed under the Creative Commons Attribution License,
which permits unrestricted use, distribution, and reproduction in any medium, provided the original work is properly cited.

\begin{abstract}
Microbial biofilms pose a serious threat to patients requiring medical devices (MDs). Prolonged periods of implantation carry a high risk of device-related infections (DRIs). Patients with DRIs often have negative outcomes following the failure of antibiotic treatment. Resistant DRIs are mainly due to the MDs contamination by bacteria producing biofilm. The present study aimed to detect biofilm formation among MD bacterial isolates and to explore their antibiotic resistance profile. The study was conducted on 76 MDs, collected at University Hospital of Campania "Luigi Vanvitelli," between October 2019 and September 2020. Identification of isolates and antibiotic susceptibility testing were performed using Matrix Assisted Laser Desorption IonizationTime of Flight Mass Spectrometry (MALDI-TOF MS) and Phoenix Becton Dickinson, respectively. Biofilm-forming abilities were assessed using the tissue culture plate (TCP) method. Among the 94 MDs isolated strains, $42.7 \%$ were Gram-positive, $40.3 \%$ Gram-negative, and 17\% Candida species. Among 78 bacterial strains, $43.6 \%$ were non-biofilm producers while $56.4 \%$ produced biofilms. All biofilm producing isolates were sensitive to a limited spectrum of antibiotic classes. All moderate and strong biofilm producers and $81 \%$ of weak biofilm producers were Multidrug Resistance (MDR) strains. In contrast, among non-biofilm producers, only $11.8 \%$ were classified as MDR strains. Our results highlighted that Sulfamides and Glycopeptides for the major Gram-positive strains and Fluoroquinolones, Carbapenems, and Aminoglycosides for the most represented Gram-negative isolates could be the most suitable therapeutic choice for most biofilm-DRIs.
\end{abstract}

\section{Introduction}

Biofilm is a structured bacterial community, enclosed in a selfproduced polymeric matrix and adhered to biotic or abiotic surfaces [1]. Compared to their planktonic counterparts, biofilm-associated bacteria exhibit greater resistance to antibiotic agents [2]. This increased antibiotic resistance is mainly due to the limited diffusion of drugs through the biofilm matrix and to physiological changes in bacteria due to the environmental conditions featuring the biofilm [3]. Furthermore, the physical proximity of the cells in the biofilm favors the acquisition of resistance through genetically transmissible elements [4]. Several pieces of evidence revealed a positive correlation between biofilm formation and the development of antibiotic resistance [5]. Abidi et al. showed that biofilm production was higher among MDR Pseudomonas aeruginosa ( $P$. aeruginosa) strains than non-MDR strains [6]. Moreover, Qayoom et al. found that the Acinetobacter baumannii (A. baumannii) MDR produced more biofilm than the non-MDR ones [7]. Regarding Staphylococcus aureus (S. aureus), Manandhar et al. associated biofilm production with Methicillin resistance $[8,9]$.

Biofilms readily develop in MDs, widely used in almost all areas of medicine for diagnostic and therapeutic 
processes and for the management of critically ill patients [1]. Prolonged use of MDs in hospitalized patients leads to a high risk of bacterial and fungal infections, defined as DRIs [10]. DRIs occur in patients with MDs in use for at least 48 hours before the infection begins $[11,12]$. These infectious diseases are feared, causing a significant morbidity and mortality and an increase of healthcare costs [13]. According to data from the Centers for Disease Control and Prevention (CDC), of nearly 2 million nosocomial infections, 50-70\% were related to MD [14]. Mortality attributed to DRIs is strongly associated with the type of device, ranging from $<5 \%$ for devices such as Foley catheters to $>25 \%$ for central venous catheters (CVCs) [14]. The three most common DRIs are centerline-associated bloodstream infection (CRBSIs), ventilator-associated pneumonia, and Foley catheter-associated urinary tract infection [15]. Among these infections, CRBSIs are highly prevalent [16]. Among these infections, CRBSIs are highly prevalent, detecting approximately 80,000 cases annually in US intensive care units. Gram-positive and Gram-negative bacteria and fungi contribute to the emergence of DRIs. The most frequently encountered strains include coagulase-negative staphylococci (CoNS), Enterococcus faecalis (E. faecalis), Klebsiella pneumoniae (K. pneumoniae), A. baumannii, P. aeruginosa, and Candida albicans [7]. Among these, S. aureus and Staphylococcus epidermidis (S. epidermidis) are estimated to cause approximately $50-70 \%$ of catheter biofilm infections $[17,18]$. The main concern about DRIs is represented by the difficulty in their eradication, due to the high antibiotic resistance of MD biofilm-associated bacteria [19]. Antibiotic therapy often unsuitable for the treatment of these infections, due to the resistant nature of the biofilm, promotes the development of serious clinical complications among DRI patients [4]. This scenario highlights the need to better understand the device-related bacteria, in order to improve the management and treatment of DRIs [20, 21]. Therefore, the present study aimed to detect the presence of biofilmforming isolates from different $\mathrm{MDs}$ and to explore their antibiotic resistance pattern. Knowledge of the main MD strains and related antibiotics susceptibility profile is essential to allow the optimal choice of antibiotic therapy for DRIs.

\section{Materials and Methods}

2.1. Sample Collection. In this study $76 \mathrm{MDs}$ were collected at the University Hospital of Campania "Luigi Vanvitelli" (Naples, Italy) from October 2019 and September 2020. Devices included 65 CVCs, 8 Foley catheters, 2 nephrostomy tubes, and 1 abdominal drain tube (Table 1). The MDs were delivered to the bacteriology laboratory and then processed.

2.2. MDs Culture and Isolate Identifications. Each MD was cut into two pieces, using sterile forceps. One piece was rolled onto the surface of a Columbia agar plate supplemented with $5 \%$ sheep blood (Oxoid, Cheshire, UK) in the presence of $\mathrm{CO}_{2}$. The other part was placed in $10 \mathrm{~mL}$ of Brain Heart Infusion broth and incubated for 24 hours. Broth was
TABle 1: Medical devices distribution.

\begin{tabular}{lc}
\hline MDs & $n(\%)$ \\
\hline Venous catheter (CVC) & $65(85,6)$ \\
Nephrostomy tube & $2(2,6)$ \\
Abdominal drain tube & $1(1,3)$ \\
Foley's catheter tip & $8(10,5)$ \\
Total & $76(100)$ \\
\hline
\end{tabular}

inoculated on CNA blood, MacConkey, Sabouraud Glucose, and Chocolate agar medium (Oxoid, Cheshire, UK). All plates were assessed after $\mathrm{O} / \mathrm{N}$ incubation at $37^{\circ} \mathrm{C}$ and further incubated for 48 hours if growth was not obvious. In positive cases, identification and antimicrobial sensitivity tests were performed. Bacterial identifications were conducted through MALDI-TOF MS (Bruker Dal-tonics, Germany). A colony from a culture agar plate was plotted on a MSP 96 MALDI-TOF (Bruker Dal-tonics, Germany), treated with $1 \mu \mathrm{L}$ of matrix solution (saturated solution of alpha-cyano-4-hydroxycinnamic acid in 50\% of acetonitrile and $2.5 \%$ of trifluoroacetic acid) (Bruker Dal-tonics, Germany) and dried for 2 minutes. The obtained spectra were imported into MALDI BioTyper 3.0 software (Bruker Daltonics, Bremen, Germany) and evaluated through standard pattern matching with respect to the main spectra. A score greater than or equal to 2.0 was associated with species identification $[22,23]$.

2.3. Antimicrobial Susceptibility Testing. The Phoenix BD (Becton Dickinson, United States) was used to confirm the identification of strains obtained via MALDI-TOF MS and to perform antibiotic susceptibility tests. Briefly, the identification broth (ID) was inoculated with pure bacterial colonies and adjusted to $0.5 \mathrm{McF}$ arland, using a Phoenix spectrophotometer. Phoenix AST broth was complemented with a drop of Phoenix AST indicator and, after that, a $25 \mu \mathrm{l}$ volume of bacterial suspension was added. ID and AST broth were loaded into the Phoenix panels, which were deposited in the Phoenix device. The results were interpreted using Epicenter software version 7.22 A (Becton Dickinson Diagnostic Systems, USA) after 16 hours of incubation [24]. The tested antibiotics in this study were ampicillin, amoxicillin/clavulanic acid, amikacin, cefotaxime, cefuroxime, fosfomycin, gentamicin, imipenem, levofloxacin, trimethoprim/sulfamethoxazole, tobramycin, piperacillin, piperacillin/tazobactam, cefotaxime, clindamycin, oxacillin, and daptomycin. Interpretative breakpoints for susceptibility and resistance were in accordance with EUCAST guidelines 2021 [25]. Resistance greater than or equal to 3 antibiotic classes defined the bacteria as MDR strain [26].

2.3.1. Biofilm Formation Assays. Biofilm formation was assessed by TCP method. Shortly, bacterial cells were inoculated in Luria Bertani (LB) broth supplemented with 1\% glucose and incubated for $24 \mathrm{~h}$ at $37^{\circ} \mathrm{C}$. After incubation, the cultures were adjusted to $\mathrm{OD}_{600 \mathrm{~nm}}$ of $0.2\left(\sim 10^{8} \mathrm{CFU} / \mathrm{mL}\right)$ with fresh LB medium with glucose. From standardized bacterial suspensions, a volume of $100 \mu \mathrm{L}$ of culture was 
inoculated into wells of a flat bottom (96-well, Thermo Fisher Scientific, Massachusetts, USA) and incubated at $37^{\circ} \mathrm{C}$ for 24 hours, statically. LB without cells were used to check sterility of media. S. aureus ATCC 6538 and S. aureus ATCC 25923 were used as negative and positive controls for biofilm production, respectively. After incubation, the biofilm was washed twice with $1 \mathrm{X}$ phosphate buffered saline (1X PBS) to remove free floating planktonic bacteria and air-dried. The wells were stained with $0.1 \%$ Crystal Violet (CV) (SigmaAldrich, St. Louis, MO) for $40 \mathrm{~min}$ at room temperature and washed three times with 1X PBS to remove excess dye. The remaining CV was solubilized by incubating with 95\% ethanol for $20 \mathrm{~min}$ under orbital shaking at room temperature. Biofilm biomass was detected by measuring the absorbance at $570 \mathrm{~nm}$, using microplate reader TECAN (Sunrise, Delaware, USA). Biofilm production was classified as negative, weak, moderate, and strong based on the cutoff value, calculated according to the following formula, using the optical density $(\mathrm{OD})$ values [27]:

ODcutoff $=$ ODavg of negative control $+(3 \times$ standard deviation of ODs of negative control).

The used criteria were as follows:

(i) OD $\leq$ ODcutoff $=$ Non-biofilm former

(ii) ODcutoff $<$ OD $\leq 2 \times$ ODcutoff $=$ Weak biofilm former

(iii) $2 \times$ ODcutoff $<$ OD $\leq 4 \times$ ODcutoff $=$ Moderate biofilm former

(iv) OD $>4 \times$ ODcutoff $=$ Strong biofilm former

All assays were performed in triplicate.

2.4. Biofilms Visualization by Scanning Electron Microscopy (SEM). The biofilms were grown on stainless steel coupons placed in a 12-well polypropylene microplate (Microtech, Naples, Italy). A volume of $1 \mathrm{~mL}$ of bacterial suspension at a density of $1 \times 10^{8} \mathrm{CFU} / \mathrm{mL}$ was added to each selected well and the plate was statically incubated for 24 hours at $37^{\circ} \mathrm{C}$. After that, the coupons were washed with 1X PBS, air-dried, and treated with plasma for 0,3 , and $30 \mathrm{~min}$. Samples were fixed in glutaraldehyde $(2.5 \% v / v)$ for 30 minutes and then dehydrated with cold solutions of ethanol at increasing concentrations $(30,50,70,90,95$, and $100 \% v / v)$, each for 20 minutes. All samples were dried in a critical point desiccator (Emitech K850, Kent, UK). Then, about 15-20 nm gold spray coating was performed with the Balzers SCD 030 (New York, USA) and the images were achieved using the Supra 40 ZEISS (EHT $=5.00 \mathrm{kV}, \mathrm{WD}=22 \mathrm{~mm}$, detector in the lens) (Berlin, Germany) [28].

2.5. Statistical Analysis. Statistical analysis was conducted using the IMB SPSS software (version 22.0; IBM SPSS Inc., New York, USA). Descriptive statistics were performed for medical device distribution and collected isolates. The antibiotic susceptibility profile of bacterial strains was expressed in percentage. Fisher's test was used to evaluate the relation between two groups of categorical variables. A $p$ value of greater than or equal to 0.05 was considered statistically significant $[29,30]$.

\section{Results}

3.1. Prevalence of Microbial Contamination of MDs. From 76 MDs, 94 strains were isolated, of which $42.7 \%$ (40) were Gram-positive, $40.3 \%$ (38) were Gram-negative, and $17 \%$ (16) were Candida species. Among Gram-positive bacteria, CoNS strains were the most frequently detected isolate (33\%), followed by E. faecalis (3.2\%) and Enterococcus faecium (E. faecium) (3.2\%), Corynebacterium striatum (C. striatum) (1.1\%), Staphylococcus aureus (S. aureus) (1.1\%), and Streptococcus agalactiae (S. agalactiae) (1.1\%). On the other hand, most representative Gram-negative bacterial strains were $P$. aeruginosa $(13.7 \%)$, succeeded by K. pneumoniae (11.6\%), Escherichia coli (E. coli) (4.3\%), A. baumannii (3.2\%), Proteus mirabilis (P. mirabilis) (3.2\%), Klebsiella aerogenes (K. aerogenes) (3.2\%), Burkholderia cepacia (B. cepacia) (1.1\%), and Serratia marcescens (S. marcescens) (1.1\%) (Figure 1). Table 2 shows that CVCs were mainly colonized by CoNS strains (42.0\%), followed by Candida species (21.7\%), P. aeruginosa (14.5\%), and K. pneumoniae (10.1\%), A. baumannii (4.4\%), P. mirabilis (2.9\%), Enterococcus species (2.9\%), and E. coli (1.5\%). Bacteria isolated in the nephrostomy tubes were Enterococcus spp. (25\%), CoNS strains (25\%), K. pneumoniae (25\%), and E. coli (25\%). In the abdominal drain tube K. pneumoniae (50\%) and Enterococcus spp. were detected. Moreover, Foley's catheter tips were mainly colonized by $P$. aeruginosa $(25 \%)$, succeeded by Enterococcus species (16.7\%), K. pneumoniae (16.7\%), and E. coli (16.7\%), CoNS strains (8.3\%), P. mirabilis (8.3\%), and Candida species $(8.3 \%)$. The current analysis revealed that some devices were colonized by more than one microorganism. The monomicrobial contaminations accounted for $77.6 \%$, while polymicrobial growths were detected in $22.4 \%$ of devices with 19.8 and $2.6 \%$ for bimicrobial and trimicrobial contaminations, respectively. Fungal/bacterial and bacterial/ bacterial copresence were each associated with a prevalence of 41.2 and $58.8 \%$. The devices subject to the greatest polymicrobial contamination were CVCs, showing the copresence of Candida species with CoNS, Enterococcus strains, $P$. aeruginosa, S. agalactiae, and K. pneumoniae. In contrast, in the trimicrobial contaminations only bacterial species were present (Figure 2).

\subsection{Prevalence of Antimicrobial Resistance among Bacteria} Isolated from $M D s$. In this study, the antimicrobial resistance profiles of S. aureus, CoNS, C. striatum, S. agalactiae, E. faecium, E. faecalis, A. baumannii, E. coli, K. pneumoniae, $P$. aeruginosa, $K$. aerogenes, $B$. cepacia, and $P$. mirabilis were evaluated. The antimicrobial resistance patterns of Grampositive and Gram-negative strains are shown in Tables 3 and 4, respectively. Among Gram-positive bacteria, CoNS strains were the most frequent, exhibiting more than $60 \%$ resistance rate to several tested antibiotics: ampicillin, ciprofloxacin, erythromycin, moxifloxacin, oxacillin, penicillin g, and rifampicin. In contrast, resistance rates to phosphomycin, 


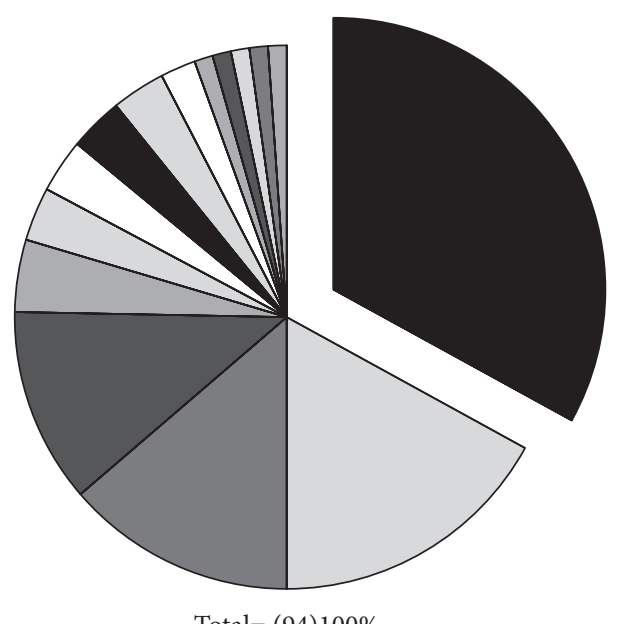

Total $=(94) 100 \%$

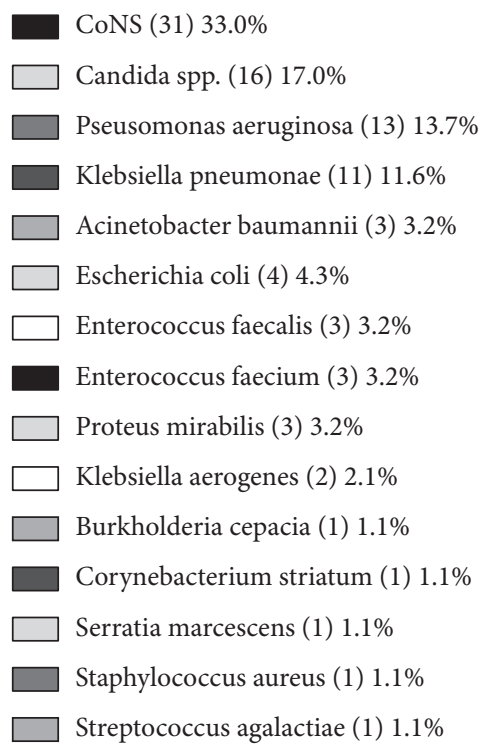

Figure 1: Prevalence of bacterial and yeast strains isolated from medical devices.

TABLE 2: Main bacterial and yeast isolates from different medical devices.

\begin{tabular}{lcccccccc}
\hline & $\begin{array}{c}\text { Enterococcus spp. } \\
(n) \%\end{array}$ & $\begin{array}{c}\text { CoNS } \\
(n) \%\end{array}$ & $\begin{array}{c}\text { K. pneumoniae } \\
(n) \%\end{array}$ & $\begin{array}{c}\text { P. aeruginosa } \\
(n) \%\end{array}$ & $\begin{array}{c}\text { E. coli } \\
(n) \%\end{array}$ & $\begin{array}{c}\text { A. baumannii } \\
(n) \%\end{array}$ & $\begin{array}{c}\text { P. mirabilis } \\
(n) \%\end{array}$ & $\begin{array}{c}\text { Candida } \\
\text { spp. } \\
(n) \%\end{array}$ \\
\hline $\begin{array}{l}\text { Venous catheter } \\
\text { Nephrostomy } \\
\text { tube }\end{array}$ & $(2) 2.9$ & $(29) 42.0$ & $(7) 10.1$ & $(10) 14.5$ & $(1) 1.5$ & $(3) 4.4$ & $(2) 2.9$ & $(15) 21.7$ \\
$\begin{array}{l}\text { Abdominal drain } \\
\text { tube }\end{array}$ & $(1) 25.0$ & $(1) 25.0$ & $(1) 25.0$ & $(0) 0.0$ & $(1) 25.0$ & $(0) 0.0$ & $(0) 0.0$ & $(0) 0.0$ \\
$\begin{array}{l}\text { Foley's catheter } \\
\text { tip }\end{array}$ & $(1) 50.0$ & $(0) 0.0$ & $(1) 50.0$ & $(0) 0.0$ & $(0) 0.0$ & $(0) 0.0$ & $(0) 0.0$ & $(0) 0.0$ \\
\hline
\end{tabular}

teicoplanin, tigecycline, and vancomycin were less than $20 \%$. Of the 31 CoNS isolates, $74.2 \%$ showed methicillin resistance phenotype and $9 \%$ macrolide-lincosamide-streptogramin B resistance phenotype. The Gram-positive strain with the highest susceptibility rate was E. faecalis; indeed, only $33.3 \%$ were resistant to Gentamicin (Table 3). Regarding Gramnegative bacteria, $P$. aeruginosa was more encountered but A. baumannii represented the most resistant strain, showing
$100 \%$ of resistance to all tested antibiotics, except for colistin. About $P$. aeruginosa, this strain had $61 \%$ resistance to fosfomycin and less than $15.8 \%$ to cefepime, ciprofloxacin, tigecycline, gentamicin, meropenem, piperacillin/tazobactam, and colistin. Of the $13 \mathrm{P}$. aeruginosa isolated, $7.7 \%$ had an extended spectrum $\beta$-lactamases-producing phenotype. Critical antibiotic resistance profile was associated with $K$. pneumoniae strains that exhibited resistance greater than 

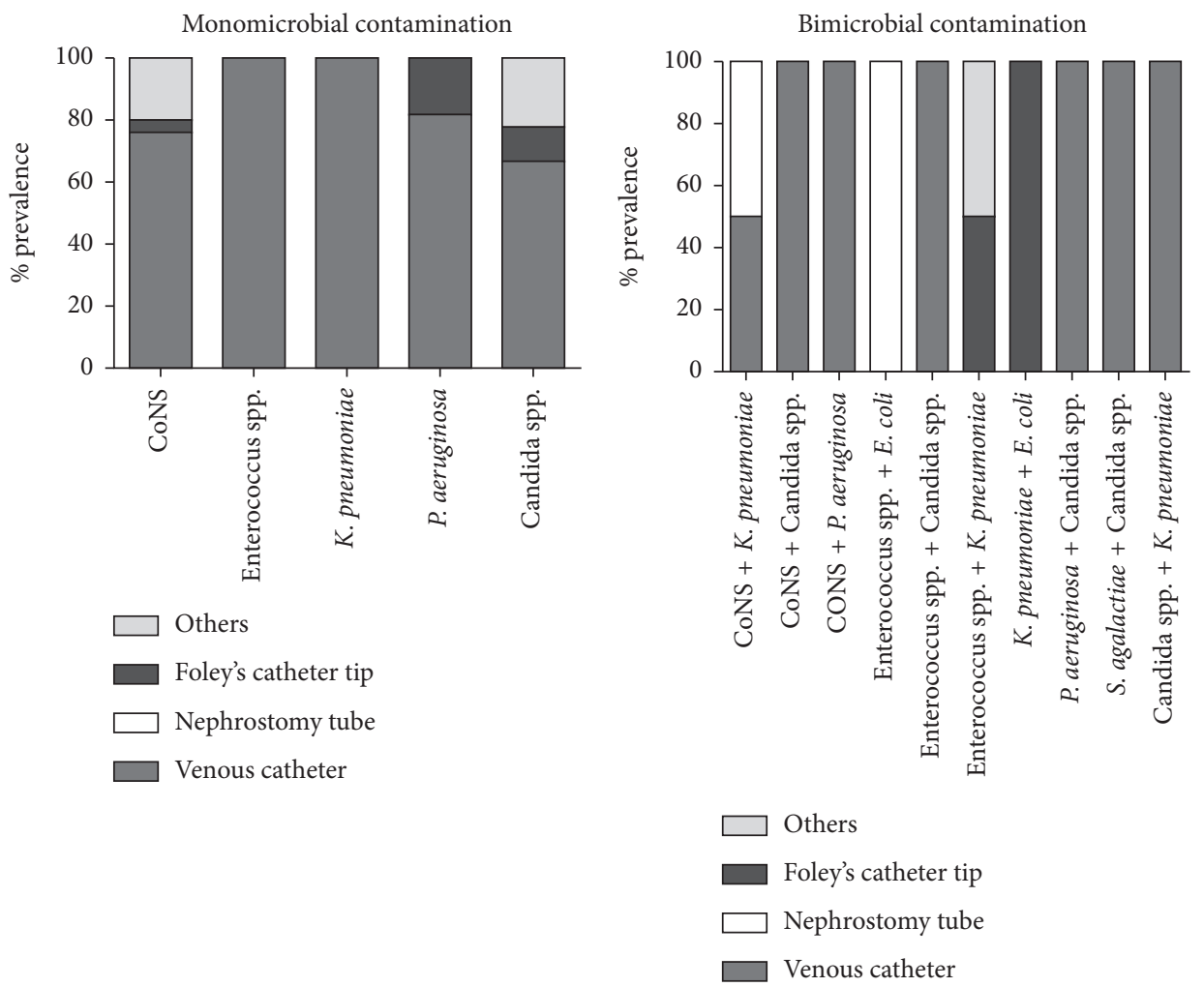

(a)

(b)

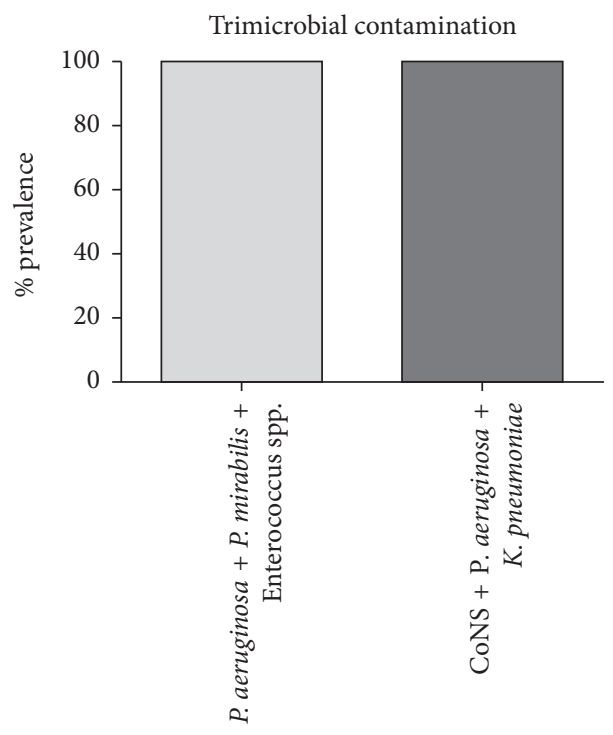

$\square$ Foley's catheter tip

$\square$ Venous catheter

(c)

FIgURE 2: Monomicrobial (a), bimicrobial (b), and trimicrobial (c) contamination of the medical devices studied.

Table 3: Antibiotic resistance profile of Gram-positive bacteria.

\begin{tabular}{lccccccccccccccccc}
\hline & \multicolumn{11}{c}{ Antibiotic resistance profile of Gram-positive bacteria (\%) } \\
& AMP & CIP & DA & DAP & E & FOS & GM & LNZ & MXF & OX & TEC & TET & TIG & PG & RIF & VA & SXT \\
\hline S. aureus & 100 & 0 & 0 & 0 & 0 & 0 & 0 & 0 & 0 & 0 & 0 & 0 & 0 & 100 & 100 & 0 & 0 \\
CoNS & 100 & 61.3 & 38.7 & 0 & 61.3 & 19.4 & 58.1 & 0 & 61.3 & 74.2 & 9.7 & 41.9 & 9.7 & 100 & 100 & 3.2 & 58.1 \\
\hline
\end{tabular}


TABLE 3: Continued.

\begin{tabular}{lccccccccccccccccc}
\hline & \multicolumn{11}{c}{ Antibiotic resistance profile of Gram-positive bacteria (\%) } \\
& AMP & CIP & DA & DAP & E & FOS & GM & LNZ & MXF & OX & TEC & TET & TIG & PG & RIF & VA & SXT \\
\hline C. striatum & - & - & 100 & - & - & - & - & 0 & 0 & - & - & 0 & - & 100 & 100 & 0 & - \\
S. agalactiae & 0 & 0 & 100 & - & 100 & - & 0 & 0 & 0 & - & 0 & 0 & - & 0 & - & 0 & - \\
E. faecium & 100 & - & - & - & - & - & 66.6 & 0 & - & - & - & 0 & 33.3 & - & - & 33.3 & - \\
E. faecalis & 100 & - & - & - & - & - & 33.3 & 0 & - & - & - & 0 & 0 & - & - & 0 & - \\
\hline
\end{tabular}

AMP: ampicillin; CIP: ciprofloxacin; DA: clindamycin; DAP: daptomycin; E: erythromycin; FOS: fosfomycin; GM: gentamicin; LNZ: linezolid; MXF: moxifloxacin; OX: oxacillin; TEC: teicoplanin; TET: tetracycline; TIG: tigecycline; PG: penicillin G; RIF: rifampicin; VA: vancomycin; SXT: trimethoprim/ Sulfamethoxazole.

TABle 4: Antibiotic resistance profile of Gram-negative bacteria.

\begin{tabular}{lccccccccccccccccc}
\hline & \multicolumn{11}{c}{ Antibiotic resistance profile of Gram-negative bacteria (\%) } \\
& AMP & AK & FEP & AMC & ERT & CAZ & CTX & IMP & CIP & TIG & FOS & GM & LEV & MEM & PIP & PIP/TAZ & CO \\
\hline A. baumannii & 100 & 100 & 0 & 0 & 100 & 0 & 0 & 100 & 100 & 0 & 0 & 100 & 100 & 100 & 0 & 0 & 0 \\
E. coli & 100 & 50 & 50 & 50 & 25 & 50 & 50 & 25 & 50 & 0 & 0 & 25 & 50 & 0 & 50 & 50 & 0 \\
K. pneumoniae & 63.6 & 18.2 & 100 & 72.7 & 63.6 & 72.7 & 81.8 & 54.6 & 0 & 45.4 & 36.4 & 54.6 & 72.7 & 63.6 & 91.0 & 63.6 & 0 \\
P. aeruginosa & 0 & 0 & 7.7 & 38.5 & 15.4 & 7.7 & 0 & 23.1 & 0 & 0 & 61.5 & 15.4 & 30.8 & 15.4 & 15.4 & 7.7 & 0 \\
K. aerogenes & 100 & 0 & 0 & 100 & 0 & 0 & 0 & 0 & 0 & 0 & 0 & 0 & 0 & 0 & 0 & 0 & 0 \\
B. cepacia & - & - & - & 100 & - & - & - & 0 & - & - & - & - & 100 & 0 & - & - & - \\
P. mirabilis & 66.6 & 0 & 66.6 & 66.6 & 0 & 66.6 & 66.6 & 0 & 66.6 & 100 & 0 & 66.6 & 66.6 & 0 & 66.6 & 0 & 100 \\
\hline
\end{tabular}

AMP: ampicillin; AK: amikacin; FEP: cefepime; AMC: amoxicillin/clavulanic acid; ERT: ertapenem; CAZ: ceftazidime; CTX: cefotaxime; IMP: imipenem; CIP: ciprofloxacin; TIG: tigecycline; FOS: fosfomycin; GM: gentamicin; LEV: levofloxacin; MEM: meropenem; PIP: piperacillin; PIP/TAZ: piperacillin/ tazobactam; CO: colistin.

$60 \%$ to ampicillin, cefepime, amoxicillin/clavulanic acid, ertapenem, ceftazidime, cefotaxime, levofloxacin, meropenem, piperacillin, and piperacillin/tazobactam (Table 4). Among K. pneumoniae isolates, $54.5 \%$ produced extended spectrum beta-lactamases, $9 \%$ showed macrolide-lincosamide-streptogramin B resistance phenotype, and $45.5 \%$ were $b_{1}{ }_{K P C}$-type carbapenemase producers. The analysis of the antibiotic susceptibility profiles of the isolated bacteria revealed that the non-MDR strains were $41.1 \%$, while the MDR ones were $58.9 \%$ ( $p$ value $<0.05$ ) (Figure 3 ).

\subsection{Detection of Biofilm Production by Bacteria Isolated from} $M D s$. In this study, strains isolated from different MDs were evaluated for their ability to produce biofilms. Of 78 bacterial strains tested, $43.6 \%$ (34) were non-biofilm producers while $56.4 \%$ (44) produced biofilms. S. agalactiae, C. striatum, E. faecalis, K. aerogenes, and B. cepacia were all non-biofilm producers. On the other side, all $A$. baumannii produced biofilms, of which $33.3 \%$ were strong biofilm producers and $66.7 \%$ moderate biofilm producers. The same biofilm production trend was detected for $P$. mirabilis. One identified strain of $S$. marcescens weakly produced biofilms. For E. coli, only $50 \%$ produced biofilms, of which $25 \%$ were moderate biofilm producers and the other $25 \%$ were weak biofilm producers. The $76.9 \%$ rate of $P$. aeruginosa was non-biofilm producers while $23.1 \%$ were biofilm producers; of the latter, 15.4 and $7.7 \%$ were moderate and weak biofilm producers, respectively. About K. pneumoniae, $72.7 \%$ were biofilms producers in which $18.2,9.1$, and $45.4 \%$ were weak, moderate, and strong producers, respectively. Among CoNS strains, 66.7\% produced biofilms, of which $32.3,22.6$, and $12.8 \%$ were strong, moderate, and weak biofilm producers, respectively (Figure 4).

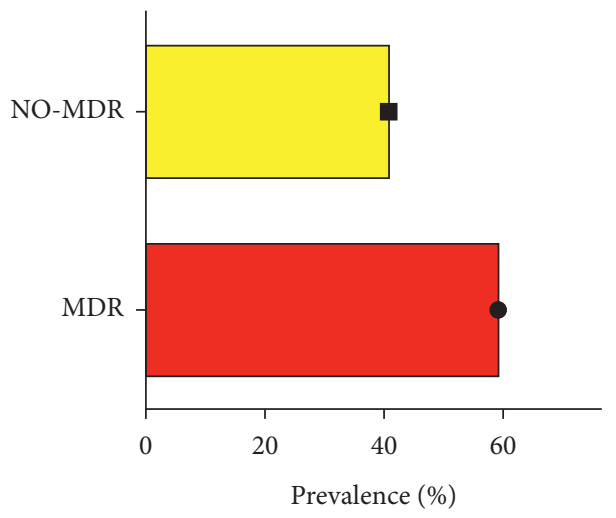

NO-MDR

MDR

FIgURE 3: Distribution of MDR and NO-MDR bacterial strains isolated from medical devices $(p$ value $<0.05$ ).

3.3.1. Biofilm Production and Antimicrobial Resistance in Bacterial Strains Isolated from MDs. The ability to secrete the biofilm matrix of MDR and non-MDR strains was assessed through the TCP method. The $\mathrm{OD}_{570 \mathrm{~nm}}$ values, as a measure of biofilm mass, related to MDR, non-MDR, and control strains, were reported in Figures 5(a) and 5(c). The absorbance values were $1.0 \pm 0.156$ and $3.62 \pm 0.517$ for the negative and positive standard strains for biofilm production, respectively. For clinical isolates, these values ranged from $6.326 \pm 0.80$ and $0.4718 \pm 0.308$. The $\mathrm{OD}_{570 \mathrm{~nm}}$ values of $54.6 \%$ (44) strains exceeded the calculated cutoff value; of these, $95.5 \%$ (42) were MDR strains and 4.5\% (2) exhibited a non-MDR phenotype. In contrast, $43.6 \%$ (34) strains were 

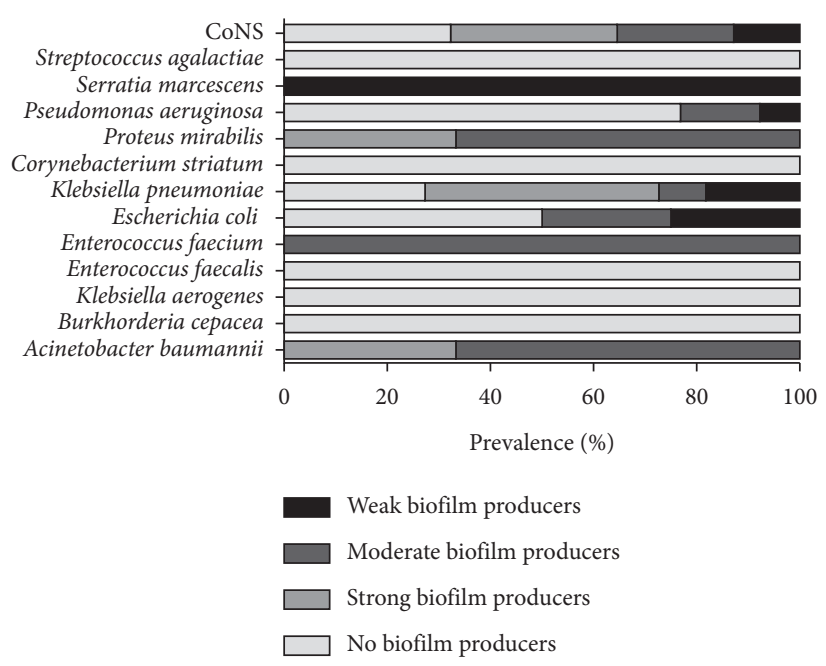

Figure 4: Biofilm formation among MD isolates.

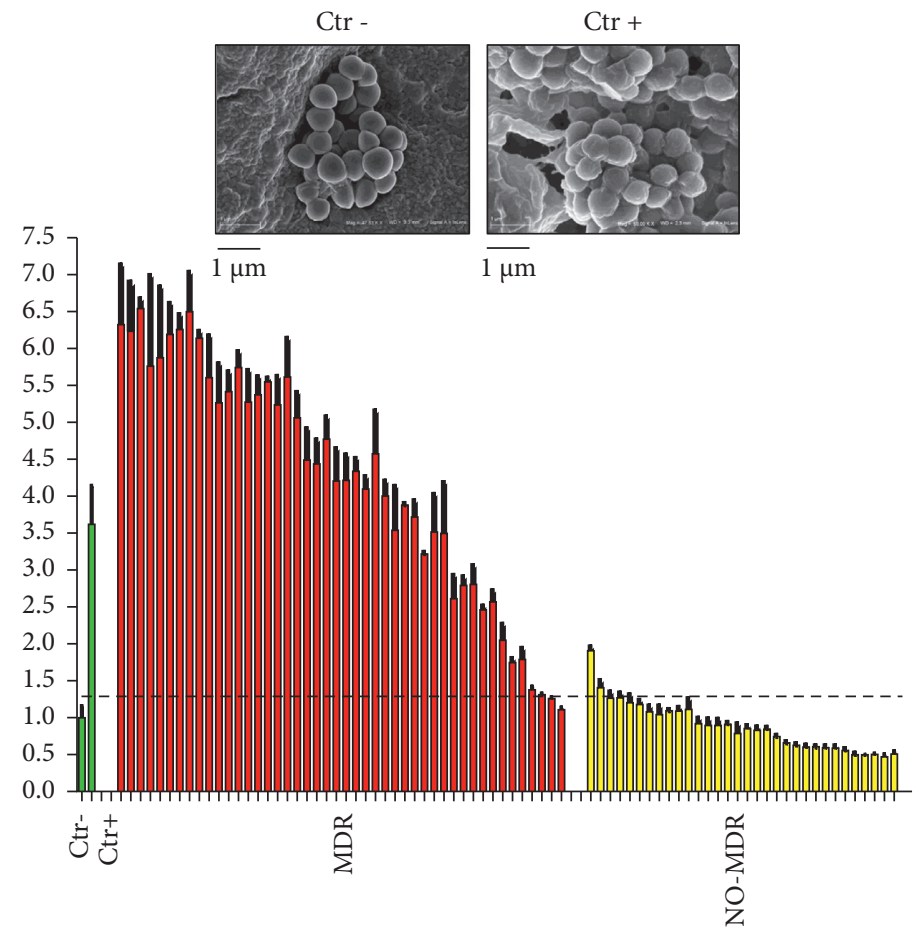

(a)

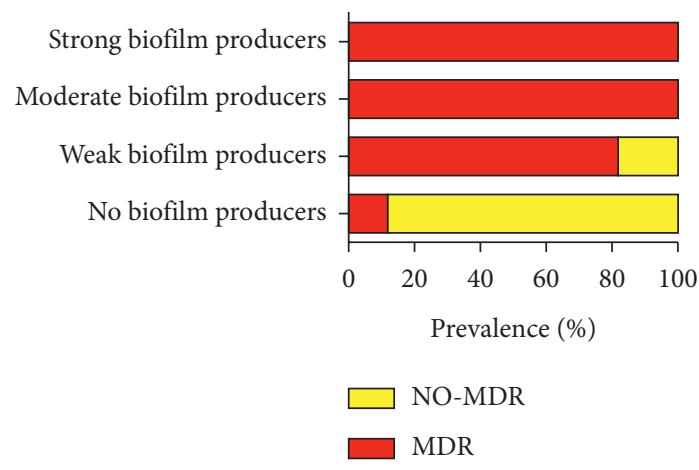

(b)

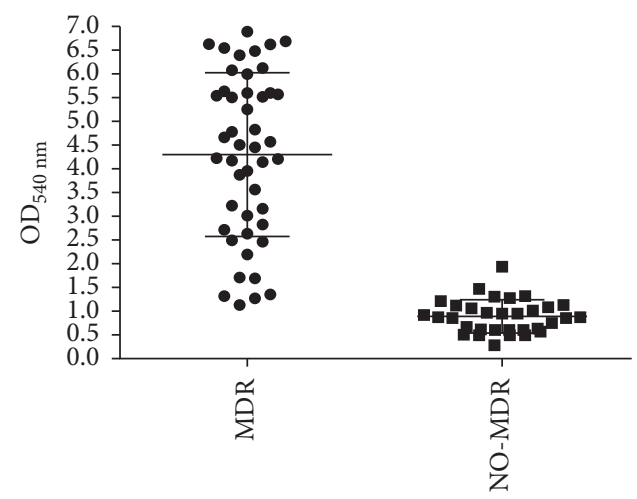

(c)

FIGURE 5: Biofilm formation analysis of MD isolates. (a) Biofilm production level of MDR, red bars, and NO-MDR, yellow bars. The green bars indicate negative and positive controls, also represented by SEM. Dashed line represents the cutoff value. (b) Prevalence of MDR and NO-MDR strains among the different biofilm productions. (c) Distribution of biofilm formation with indicated resistance phenotypes. 
associated with absorbance values below the cutoff value; of the latter $88.2 \%$ were non-MDR and $11.8 \%$ were MDR strains $(p$ value $<0.05)$. Among the non-biofilm producing strains, $11.8 \%$ were $\mathrm{MDR}$ and $88.2 \%$ non-MDR isolates. Weak biofilm producers consisted of $81.8 \%$ MDR and $18.2 \%$ non-MDR strains. Instead, all moderate and strong biofilm producers resulted as MDR strains (Figure 5(b)). These data underlined the positive correlation between biofilm formation and resistance phenotype, main cause of DRI treatment problems. The sensitivity profiles to penicillins, fluoroquinolones, cephalosporins, carbapenems, sulfonamides, aminoglycosides, macrolides, and glycopeptides of E. coli, P. aeruginosa, K. pneumoniae, and CoNS biofilm and non-biofilm producers were evaluated and compared (Figure 6). All biofilm producing isolates had a high rate of resistance to the analyzed antibiotic classes. Among E. coli, all biofilm producing strains were resistant to penicillins, fluoroquinolones, and cephalosporins, while only $50 \%$ showed sensitivity to Carbapenems. In contrast, the nonbiofilm producing counterparts were susceptible to all antibiotic classes tested (Figure 6(a)). Dwelling on the biofilm producing $P$. aeruginosa strains all exhibited resistance to Penicillins, unlike non-biofilm producers. For cephalosporins and carbapenems, biofilm-producing $P$. aeruginosa showed $50 \%$ susceptibility rates, whereas the non-biofilm producing counterparts were 100 and $81.8 \%$ sensitive, respectively. For Fluoroquinolones, a susceptibility of 100 and $72.7 \%$ was detected for producing and nonproducing biofilm, respectively (Figure 6(b)). Biofilm-producing K. pneumoniae exhibited susceptibility levels of 75, 25, and $12.5 \%$ to aminoglycosides, sulfonamides, and carbapenems, respectively. In contrast, all biofilm producing $K$. pneumoniae strains were resistant to Fluoroquinolones and Cephalosporins. Sensitivity of $100 \%$ to all tested antibiotic classes was found for non-biofilm producing strains. Significant variations in sensitivity levels of biofilm producers and nonproducers to sulfonamides, carbapenems, fluoroquinolones, and cephalosporins were detected $(p \quad$ value $<0.05)$ (Figure 6(c)). Regarding biofilm producing CoNS strains, they exhibited $100 \%$ resistance to penicillins and susceptibility rates of $96.8,21.7,26,52.5$, and $56.2 \%$ to glycopeptides, fluoroquinolones, aminoglycosides, macrolides, and sulfonamides, respectively. In contrast, all non-biofilm producing CoNS strains were susceptible to glycopeptides, while the sensitivity rates ranging from 55.5 to $88.8 \%$ were found for fluoroquinolones, aminoglycosides, macrolides, and sulfonamides. Significant differences were encountered between the sensitivity levels of biofilm producing and non-biofilm producing strains to aminoglycosides, fluoroquinolones, and penicillins $(p$ value $<0.05)$ (Figure 6(d))

\section{Discussion}

Extensive and prolonged use of MDs is associated with a significant risk of infectious complications that prolong hospitalization and raise healthcare costs [10]. Most patients with DRIs have a negative outcome, following antibiotic therapy failure. Resistant DRIs are mainly due to complex characteristics of bacterial biofilms, such as the shielding effect of the biofilm matrix which limits the penetration of antibiotic, the physical proximity of bacterial cells which promotes the exchange of resistance gene elements, and the slow cell growth rate [31]. Therefore, the present study defines the main pathogens that contaminate MDs, their propensity to form biofilms, and the related patterns of antibiotics susceptibility, to improve the management and treatment of DRIs. To conduct this analysis, 94 strains were isolated from CVC, Foley catheters, nephrostomy, and abdominal drain tube over a period of approximately 1 year. Among these isolates, $42.7 \%$ were Gram-positive, $40.3 \%$ were Gram-negative, and $17 \%$ were Candida species. About the Gram-negative bacteria, P. aeruginosa, K. pneumoniae, and E. coli were the most prominent, while, among Grampositive bacteria, CoNS species represented the most isolated strains. The mainly isolated MDs strains belonged to normal commensal flora or were of nosocomial origin [32]. The highest incidence of Gram-positive strains (44.9\%) was detected in the CVC samples, in accordance with other studies. Sohail and Latif revealed that $64 \%$ of CVCs were colonized by Gram-positive bacteria, $26 \%$ by Gram-negative bacteria, and $10 \%$ by Candida species [33]. Likewise, Lombardi et al. reported that 54\% of CVCs were colonized by Gram-positive bacteria [34]. Our data reported that CoNS strains contributed most to the contamination of CVCs, as reported by Lombardi et al. [34]. In contrast the study conducted in Pakistan identified $S$. aureus as common pathogen found in these devices (39\%) [33]. A wider prevalence of Gram-negative strains (66.7\%) was detected in the Foley catheters, with a higher incidence of $P$. aeruginosa (25\%). Almalki and Varghese detected 89\% of Gram-negative bacteria on the same devices, of which $E$. coli was the most frequently encountered (26\%) [35].

Analysis of the antibiotic susceptibility profiles of all isolated strains allowed classifying the bacteria in MDR and NO-MDR strains. Our data detected 59.2 and $40.8 \%$ of MDR and NO-MDR strains, respectively. These strains were evaluated for their propensity to produce biofilms. Of the 78 bacterial strains investigated, $56.4 \%$ were biofilm producers. The major producers were A. baumannii (100\%), S. marcescens (100\%), and P. mirabilis (100\%), K. pneumoniae (72.7\%), CoNS strains (67.7\%), and E. coli (50\%). Similar data were reported in the study of Revdiwala et al., in which the largest biofilm producers isolated from MDs were CoNS strains (88.9\%), K. pneumoniae (100\%), E. coli (68.8\%), and A. baumannii (95.3\%) [36]. The population showing biofilm production contained a high percentage of isolated MDR. Indeed, all moderate and strong biofilm producers and $81.8 \%$ of weak biofilm producers were MDR strains. Several studies revealed a positive correlation between biofilm formation and the development of antibiotic resistance [37]. In particular, Abidi et al. showed that biofilm production was higher among MDR $P$. aeruginosa strains than in non-MDR strains [6]. Moreover, Amin et al. found that the A. baumannii MDR strains produced more biofilm than the non-MDR ones [7]. The association between the MDR phenotype and biofilm production underlined the need to better investigate the resistance profiles of biofilm producing strains. Bacterial resistance 


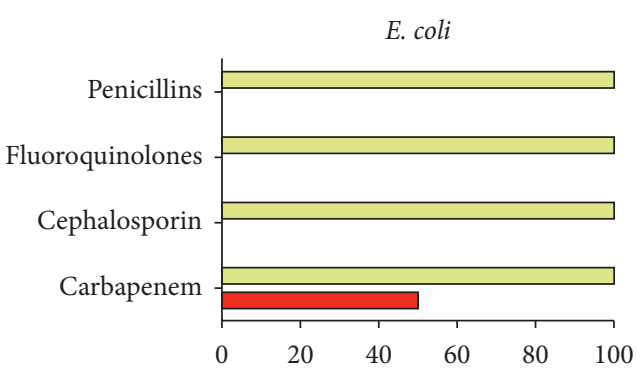

(a)

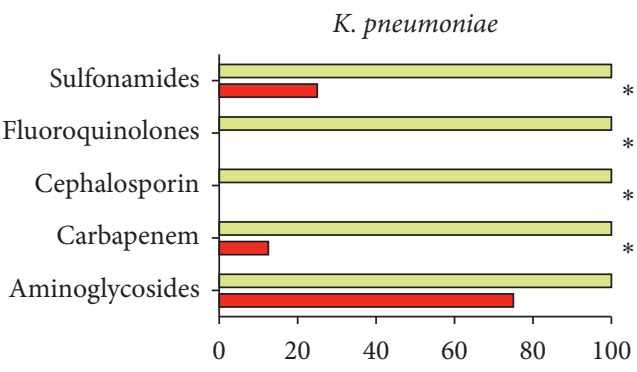

(c)

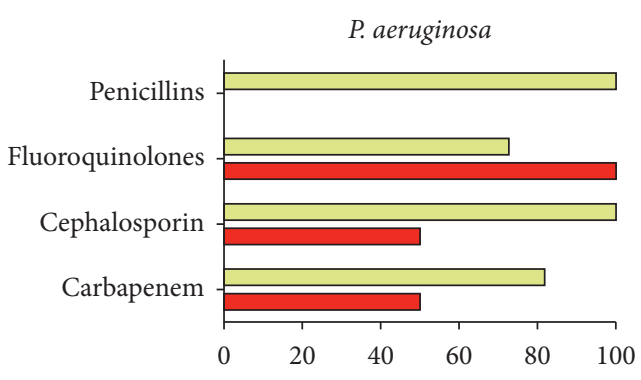

(b)

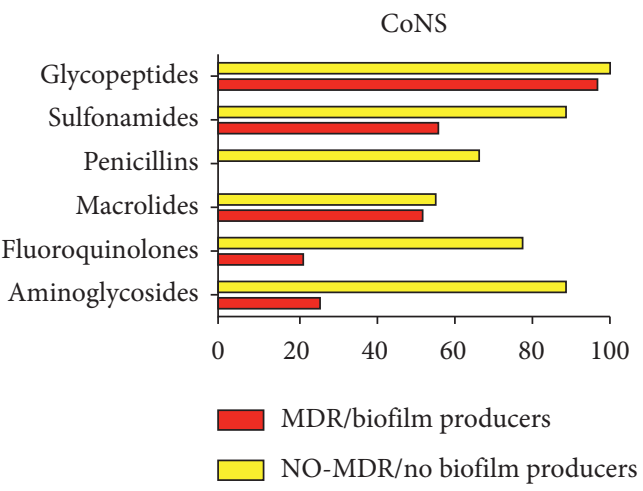

(d)

Figure 6: Susceptibility rate to different classes of antibiotics in E. coli (a), P. aeruginosa (b), K. pneumoniae (c), and CoNS (d) between biofilm and non-biofilm producers. ${ }^{*} p$ value $<0.05$.

patterns showed that E. coli biofilm producing strains exhibited $100 \%$ resistance to Penicillins, Fluoroquinolones, and Cephalosporins and 50\% sensitivity to Carbapenems. Our data showed that Carbapenems could be efficient for the antibiotic treatment of DRIs caused by E. coli biofilm producing strains. In agreement with our study, Penicillin resistance of approximately $90 \%$ was reported in a study conducted in Nepal by Neupane et al. In contrast to our data, they noted resistance rates of 58.3 and $50 \%$ related to Fluoroquinolones and Cephalosporins [38]. Regarding $P$. aeruginosa, the biofilm producing strains were all resistant to Penicillins, while they were sensitive to cephalosporins (50\%), carbapenems (50\%), and fluoroquinolones (100\%). The sensitivity levels of these biofilm producing strains indicated fluoroquinolones as a potential treatment for DRIs due to $P$. aeruginosa. Our data agreed with the study of Abidi et al., reporting that $P$. aeruginosa biofilm producing strains exhibited lowest resistance against fluoroquinolones and cephalosporins [6]. Concerning K. pneumoniae, all biofilm producing strains were resistant to fluoroquinolones and cephalosporins. Susceptibility rates below $75 \%$ were recorded for sulfonamides, carbapenems, and aminoglycosides. The high sensitivity rates to aminoglycosides indicated that it could be used for treatment of $K$. pneumoniae biofilm producing strains. Our data was in contrast with a study conducted in Indonesia, which attributed a resistance rate higher than 70.83 to Aminoglycosides and lower than $1.40 \%$ to carbapenems [39]. About CoNS biofilm producing strains, they showed susceptibility levels higher than $56.2 \%$ to sulfonamides and glycopeptides and lower than $52.2 \%$ to macrolides, fluoroquinolones, and aminoglycosides. Due to the increased susceptibility levels to glycopeptides and sulfonamides, they could be selected as a potential treatment for DRIs caused by CoNS biofilm producing strains. Similar data were obtained from a study conducted by Shresthe et al. that reported $100,78.3$, and $3.2 \%$ of CoNS biofilm producing strains resistant to penicillins, fluoroquinolones, and glycopeptides, respectively. In contrast, they listed resistance levels of 66,80 , and $50 \%$ for macrolides, sulfonamides, and aminoglycosides, respectively [40]. The reported low levels of antibiotic susceptibility reflect a worrying reality associated with the DRIs. Several studies reported similar conditions in other contexts, highlighting the generalized spread of MDs-related resistances [41]. Currently, there are no guidelines for the clinicians to treat DRIs, although they are associated with untreatable cases [42]. We suggest that empirical antibiotic treatment should be based on localized epidemiological trend data. Our study provides information on the current situation in our University Hospital, to define novel guidelines for the correct treatment of DRIs.

\section{Conclusions}

In the present study, the worrying prevalence of MDR biofilm producing strains represents a serious challenge to clinicians in the treatment and care of hospitalized patients. Antibiotics belonging to the class of glycopeptides and sulfonamides for CoNS strains and fluoroquinolones, carbapenems, and aminoglycosides for P. aeruginosa, E. coli, and $K$. pneumoniae isolates were found to be more effective 
for most biofilm producing strains. A better understanding of the biofilm producing strains isolated from MDs and related antibiotic resistance profiles could help define a more effective treatment plan to improve patient management and stimulate the scientific community to search for novel treatment strategies to combat this real threat [43].

\section{Data Availability}

No data were used to support this study.

\section{Conflicts of Interest}

The authors declare no conflicts of interest.

\section{Authors' Contributions}

Veronica Folliero and Gianluigi Franci have contributed equally.

\section{Acknowledgments}

The authors would like to thank the staff of University Hospital of Campania "Luigi Vanvitelli" for their contributions.

\section{References}

[1] L. K. Vestby, T. Grønseth, R. Simm et al., "Bacterial biofilm and its role in the pathogenesis of disease," Antibiotics, vol. 9, no. 2, p. 59, 2020.

[2] G. Gebreyohannes, A. Nyerere, C. Bii et al., "Challenges of intervention, treatment, and antibiotic resistance of biofilmforming microorganisms," Heliyon, vol. 5, no. 8, Article ID e02192, 2019.

[3] G. Franci, V. Folliero, M. Cammarota et al., "Epigenetic modulator UVI5008 inhibits MRSA by interfering with bacterial gyrase," Scientific Reports, vol. 8, p. 13117, 2018.

[4] D. Sharma, L. Misba, A. U. Khan et al., "Antibiotics versus biofilm: an emerging battleground in microbial communities," Antimicrobial Resistance and Infection Control, vol. 8, p. 76, 2019.

[5] L. Qi, H. Li, C. Zhang et al., "Relationship between antibiotic resistance, biofilm formation, and biofilm-specific resistance in Acinetobacter baumannii," Frontiers in Microbiology, vol. 7, p. $483,2016$.

[6] S. H. Abidi, S. K. Sherwani, T. R. Siddiqui et al., "Drug resistance profile and biofilm forming potential of Pseudomonas aeruginosa isolated from contact lenses in KarachiPakistan,” BMC Ophthalmology, vol. 13, p. 57, 2013.

[7] S. Qayoom, M. Amin, V. Pai et al., "Biofilm formation and multidrug resistance in nosocomial isolates of Acinetobacter," IJMR, vol. 5, no. 3, pp. 425-429, 2020.

[8] S. Manandhar, A. Singh, A. Varma et al., "Biofilm producing clinical Staphylococcus aureus isolates augmented prevalence of antibiotic resistant cases in tertiary care Hospitals of Nepal," Frontiers in Microbiology, vol. 9, p. 2749, 2018.

[9] D. Pignataro, F. Foglia, M. T. Della Rocca et al., "Methicillinresistant Staphylococcus aureus: epidemiology and antimicrobial susceptibility experiences from the university hospital "luigi Vanvitelli" of Naples," Pathogens and Global Health, vol. 114, no. 8, pp. 451-456, 2020.
[10] Z. Khatoon, C. D. McTiernan, E. J. Suuronen et al., "Bacterial biofilm formation on implantable devices and approaches to its treatment and prevention," Heliyon, vol. 4, no. 12, Article ID e01067, 2018.

[11] V. Folliero, P. Caputo, M. T. Della Rocca et al., "Prevalence and antimicrobial susceptibility patterns of bacterial pathogens in urinary tract infections in University Hospital of Campania "Luigi Vanvitelli" between 2017 and 2018," Antibiotics, vol. 9, no. 5, p. 215, 2020.

[12] M. Haque, M. Sartelli, J. McKimm et al., "Health care-associated infections - an overview," Infection and Drug Resistance, vol. 11, pp. 2321-2333, 2018.

[13] T. Olsen, O. D. Jørgensen, J. C. Nielsen et al., "Incidence of device-related infection in 97750 patients: clinical data from the complete Danish Device-Cohort (1982-2018)," European Heart Journal, vol. 40, no. 23, pp. 1862-1869, 2019.

[14] J. S. Van Epps and J. G. Younger, "Implantable device-related infection," Shock, vol. 46, no. 6, pp. 597-608, 2016.

[15] S. D Advani, R. A Lee, M. Long et al., "The impact of 2015 NHSN catheter-associated urinary tract infection (CAUTI) definition change on central line-associated bloodstream infection (CLABSI) rates and CLABSI prevention efforts at an academic medical center," Infection Control and Hospital Epidemiology, vol. 39, no. 7, pp. 878-880, 2018.

[16] https://www.cdc.gov/infectioncontrol/guidelines/bsi/ introduction.html.

[17] Y. Zheng, L. He, T. K Asiamah et al., "Colonization of medical devices by Staphylococci," Environmental Microbiology, vol. 20, no. 9, pp. 3141-3153, 2018.

[18] W. F. Oliveira, P. M. S. Silva, R. C. S. Silva et al., "Staphylococcus aureus and Staphylococcus epidermidis infections on implants," Journal of Hospital Infection, vol. 98, no. 2, pp. 111-117, 2018.

[19] J. W. Costerton, P. S. Stewart, and E. P. Greenberg, "Bacterial Biofilms: a common cause of persistent infections," Science, vol. 21, no. 5418, pp. 1318-1322, 1999.

[20] A. B. Eyoh, M. Toukam, J. Atashili et al., "Relationship between multiple drug resistance and biofilm formation in Staphylococcus aureus isolated from medical and nonmedical personnel in Yaounde, Cameroon," Pan African Medical Journal, vol. 17, p. 186, 2014.

[21] S. Hogan, N. T. Stevens, H. Humphreys et al., "Current and future approaches to the prevention and treatment of Staphylococcal medical device-related infections," Current Pharmaceutical Design, vol. 21, no. 1, pp. 100-113, 2015.

[22] F. Martora, F. Pinto, V. Folliero et al., "Isolation, characterization and analysis of pro-inflammatory potential of Klebsiella pneumoniae outer membrane vesicles," Microbial Pathogenesis, vol. 8, no. 12, p. 19, 2019.

[23] I. Kotaskova, H. Obrucova, B. Malisova et al., "Molecular techniques complement culture-based assessment of bacteria composition in mixed biofilms of urinary tract catheter-related samples," Frontiers in Microbiology, vol. 10, p. 462, 2019.

[24] E. Serretiello, R. Astorri, A. Chianese et al., "The emerging tick-borne Crimean-Congo haemorrhagic fever virus: a narrative review," Travel Medicine and Infectious Disease, vol. 37, p. 101871, 2020.

[25] https://www.eucast.org/clinical_breakpoints/.

[26] M. Exner, S. Bhattacharya, B. Christiansen et al., "Antibiotic resistance: what is so special about multidrug-resistant Gramnegative bacteria?" GMS Hygiene Infection Control, vol. 12, p. Doc05, 2017.

[27] L. B. Shrestha, N. R. Bhattarai, and B. Khanal, "Comparative evaluation of methods for the detection of biofilm formation 
in coagulase-negative staphylococci and correlation with antibiogram," Infection and Drug Resistance, vol. 11, pp. 607-613, 2018.

[28] M. Relucenti, G. Familiari, O. Donfrancesco et al., "Microscopy methods for biofilm imaging: focus on SEM and VPSEM pros and cons," Biology, vol. 10, no. 1, p. 51, 2021.

[29] F. Petrillo, D. Pignataro, F. M. Di Lella et al., "Antimicrobial susceptibility patterns and resistance trends of staphylococcus aureus and coagulase-negative staphylococci strains isolated from ocular infections," Antibiotics, vol. 10, no. 5, p. 527, 2021.

[30] E. Serretiello, V. Folliero, B. Santella et al., "Trend of bacterial uropathogens and their susceptibility pattern: study of single academic high-volume center in Italy (2015-2019)," The Internet Journal of Microbiology, vol. 2021, Article ID 5541706, 10 pages, 2021.

[31] M. H. Muhammad, A. L. Idris, X. Fan et al., "Beyond risk: bacterial biofilms and their regulating approaches," Frontiers in Microbiology, vol. 11, p. 928, 2020.

[32] P. Singha, M. J. Goudie, Q. Liu et al., "Multi-pronged approach to combat catheter-associated infections and thrombosis by combining nitric oxide and a polyzwitterion: a 7-day in vivo study in a rabbit model," ACS Applied Materials \& Interfaces, vol. 12, no. 8, pp. 9070-9079, 2021.

[33] M. Sohail and Z. Latif, "Molecular analysis biofilm formation, and busceptibility of methicillin-resistant Staphylococcus aureus strains causing community and Health Care-Associated infections in central venous catheters," Revista da Sociedade Brasileira de Medicina Tropical, vol. 51, pp. 603609, 2018.

[34] S. Lombardi, M. Scutell, V. Felice et al., "Central vascular catheter infections in a Hospital of central Italy," New Microbiologica, vol. 37, no. 1, pp. 41-50, 2014.

[35] M. A. Almalki and R. Varghese, "Prevalence of catheter associated biofilm producing bacteria and their antibiotic sensitivity pattern," Journal of King Saud University Science, vol. 32, pp. 1427-1433, 2020.

[36] S. Revdiwala, B. M. Rajdev, and S. Mulla, "Characterization of bacterial etiologic agents of biofilm formation in medical devices in critical care setup," Critical Care Research and Practice, vol. 2012, Article ID 945805, 6 pages, 2012.

[37] S. A. Senobar Tahaei, A. Stájer, I. Barrak et al., "Correlation between biofilm-formation and the antibiotic resistant phenotype in Staphylococcus aureus isolates: a laboratory-based study in Hungary and a review of the literature," Infection and Drug Resistance, vol. 14, pp. 1155-1168, 2021.

[38] S. Neupane, N. Dutt Pant, and S. Khatiwada, "Correlation between biofilm formation and resistance toward different commonly used antibiotics along with extended spectrum beta lactamase production in uropathogenic Escherichia coli isolated from the patients suspected of urinary tract infections visiting Shree Birendra Hospital, Chhauni, Kathmandu, Nepal," Antimicrobial Resistance and Infection Control, vol. 5, p. 5, 2016.

[39] H. Nirwati, K. Sinanjung, F. Fahrunissa et al., "Biofilm formation and antibiotic resistance of Klebsiella pneumoniae isolated from clinical samples in a tertiary care hospital, Klaten, Indonesia," BMC Proceedings, vol. 13, no. 11, p. 20, 2019.

[40] L. B. Shrestha, N. R. Bhattarai, B. Khanal et al., "Antibiotic resistance and biofilm formation among coagulase-negative staphylococci isolated from clinical samples at a tertiary care hospital of eastern Nepal," Antimicrobial Resistance and Infection Control, vol. 6, no. 89, 2017.
[41] S. Dincer, F. Masume Uslu, and A. Delik, "Antibiotic resistance in biofilm," in Bacterial Biofilms, S. Dincer, M. Sümengen Özdenefe, and A. Arkut, Eds., IntechOpen, London, UK, 2020.

[42] E. Olivares, S. Badel-Berchoux, C. Provot et al., "Clinical impact of antibiotics for the treatment of Pseudomonas aeruginosa biofilm infections," Frontiers in Microbiology, vol. 10, p. 2894, 2019.

[43] M. Singh, C. Zannella, V. Folliero et al., "Combating actions of green 2D-materials on gram positive and negative bacteria and enveloped viruses," Frontiers in Bioengineering and Biotechnology, vol. 8, Article ID 569967, 2020. 